\title{
ARTÍCULOS
}

\section{AL SERVICIO DEL AMIGO AMERICANO. LA ACCIÓN EXTERIOR VASCA EN ESTADOS UNIDOS CONTRA EL FRANQUISMO (1941-1945)}

\section{Serving to American Friend. The Basque Foreign Action in the USA against Francoism (1941-1945)}

\section{David Mota Zurdo}

Univ. del País Vasco-Euskal Herriko Unibertsitatea david.mota@ehu.eus

Recibido: 20/05/2016 - Aceptado: 22/10/2016

Cómo citar este artículo/Citation:

David Mota ZURDo (2017), "Al servicio del amigo americano. La acción exterior vasca en Estados Unidos contra el franquismo (1941-1945)", Hispania Nova, 15, págs. 172 a 190, DOI: https://doi.org/10.20318/hn.2017.3485
Copyright: (c) HISPANIA NOVA es una revista debidamente registrada, con ISSN 1138-7319 y Depósito Legal M 9472-1998. Los textos publicados en esta revista están -si no se indica lo contrario- bajo una licencia Reconocimiento-Sin obras derivadas 3.0 España de Creative Commons. Puede copiarlos, distribuirlos y comunicarlos públicamente siempre que cite su autor y la revista y la institución que los publica y no haga con ellos obras derivadas. La licencia completa se puede consultar en: http://creativecommons.org/licenses/by-nd/3.0/es/deed.es
Resumen El Servicio Vasco de Información fue una organización surgida durante la Guerra Civil española, al amparo del Partido Nacionalista Vasco y del Gobierno vasco. A partir de 1937, ya en el exilio, se utilizó como instrumento para la materialización de un proyecto político del Gobierno vasco y del PNV dirigido a conseguir la ayuda de las potencias extranjeras (Francia, Gran Bretaña y Estados Unidos) para combatir al franquismo, obtener financiación para mantener activas las instituciones vascas y recuperar el autogobierno vasco. En este artículo se analizan las relaciones que mantuvo el Gobierno vasco con las agencias estadounidenses ( $\mathrm{FBI}, \mathrm{COI}, \mathrm{OSS}, \mathrm{CIAA}$, Ejército) en materia de información y propaganda durante la II Guerra Mundial, haciendo especial hincapié en las establecidas con la OSS y en las iniciativas de esta última para ayudar a la restauración democrática en España.

Palabras clave: Servicio Vasco de Información, Gobierno vasco en el exilio, Departamento de Estado de Estados Unidos, OSS, FBI, Franquismo, II Guerra Mundial.
Abstract: The Basque Intelligence Service was an organization emerged during the Spanish Civil War, under the shelter of the Basque Nationalist Party and the Basque Government. Since 1937, in exile, it was harnessed as an instrument for the materialization of a political project of the Basque Government and PNV, led to enlist the help of the foreign powers (France, Great Britain and the United States) to fight francoism, obtain funds to mantain active the Basque political institutions and to recover the Basque selfgovernment. In this study, it is analyzed the relations that kept the Basque government with US agencies (FBI, COI, OSS, CIAA, Army) on information and propaganda during World War II, with particular emphasis on those established with the OSS and the initiatives of the latter to assist the restoration of democracy in Spain.

Key words: Basque Intelligence Service, Basque Government in-exile, US State Department, OSS, FBI, Francoism, World War II. 


\section{AL SERVICIO DEL AMIGO AMERICANO. LA ACCIÓN EXTERIOR VASCA EN ESTADOS UNIDOS CONTRA EL FRANQUISMO (1941-1945)}

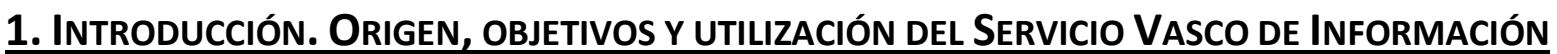

El primer Gobierno vasco, creado en octubre de 1936 en la complicada coyuntura de la Guerra Civil española (1936-1939) y presidido por el nacionalista vasco José Antonio Aguirre, se vio obligado a actuar desde su nacimiento como un Estado soberano, desplegando una acción exterior propia para hacer frente a las necesidades de la guerra (suministros, comunicaciones y asistencia a los refugiados, principalmente $)^{1}$. Para la realización de estas labores, Aguirre contó con la participación de Antón Irala, secretario de la presidencia, que se encargó de confeccionar una red de colaboradores que favorecieran las comunicaciones entre la sede del Gobierno vasco en Bilbao y la delegación vasca en Bayona ${ }^{2}$. Este grupo de confianza se creó en otoño de 1936, durante un viaje a Francia que realizó Irala para comprar armas y suministros para el ejército vasco. Semanas antes, varios de aquellos hombres, entre los que se encontraban los hermanos Agesta y Mitxelena, habían creado en la Junta local del PNV de Irún (Guipúzcoa) el germen de lo que posteriormente se denominó Servicio Vasco de Información (SVI) o Servicios. Éstos originariamente nacieron como organización auxiliar integrada en el PNV pero progresivamente pasaron a insertarse dentro del organigrama del Gobierno vasco sin perder su orientación política ${ }^{3}$.

El minucioso trabajo de los miembros del incipiente SVI, sumado a la amplia red de barcos (Txintxerpe, Domayo, Napartarra) situados en el Golfo de Vizcaya, que favorecieron las comunicaciones entre el interior y el exilio, permitió que el ejecutivo de Aguirre y su partido pudieran mantener una

\footnotetext{
1 José Luis de la GRANJA: El oasis vasco El nacimiento de Euskadi en la Il República y la Guerra Civil, Madrid: Tecnos, 2003. Alexander UGALDE: “La actuación internacional cultura e investigación vasca, no 6 (1996), pp. 187210. José Luis de la GRANJA: "El nacimiento de Euskadi: el Estatuto de 1936 y el primer gobierno vasco", Historia Contemporánea, no 35(2007) pp. 427-450. Ludger MEES y Santiago de PABLO: "El gobierno vasco en el exilio", Cuadernos de Alzate, no18 (1998), pp. 41-56.

2 Durante la Guerra Civil, el Gobierno vasco desplegó una red de delegaciones con finalidad representativa y atribuciones similares a las de una embajada de un Estado de pleno derecho. Alexander UGALDE, La acción exterior del nacionalismo vasco (1890-1936). Historia, pensamiento y relaciones internacionales, Oñati: IVAP, 1996. Óscar ÁlVAREZ GILA ET AL..: Delegaciones de Euskadi (1936-1975), Vitoria-Gasteiz: Servicio Central de Publicaciones del Gobierno Vasco, 2010. Mikel RODRÍGUEZ: Espías Vascos, Tafalla: Txalaparta, 2004, pp. 73 y ss.

3 Juan Carlos JIMÉNEZ DE ABERÁSTURI y Rafael MORENO: Al servicio del extranjero, Historia del Servicio Vasco de Información (1936-1943), Madrid: Antonio Machado Libros, 2009, pp. 42.
} 
valija diplomática periódica al establecer una red de comunicación medianamente regular entre Bilbao y la mencionada delegación vasca en Francia ${ }^{4}$.

Así las cosas, la caída del frente norte en 1937 y el obligado exilio de la dirección política vasca, provocó que el SVI cobrara especial relevancia. El Gobierno vasco y el PNV acordaron que los Servicios crearan un sistema de enlaces desde Bayona para establecer de forma continuada el contacto entre el interior y el exilio. Esta organización se conoció como red Álava, nombre que hacía alusión al apellido de su principal responsable: el nacionalista vasco Luis Álava Sautu ${ }^{5}$. En el interior, sus principales activos fueron mujeres (Delia Lauroba y Teresa Verdes, entre otras) que se encargaron de establecer una comunicación fluida con los dirigentes nacionalistas vascos encarcelados (como Juan Ajuriaguerra) y el exterior, y de obtener información sobre los motivos de la rendición del ejército vasco en Santoña (Cantabria) $)^{6}$.

Según han señalado algunos de los principales especialistas, durante los años previos a la II Guerra Mundial y posteriormente durante la contienda, el SVI se encargó fundamentalmente de la propaganda y la información, pero también del desempeño de una función política al ofrecer sus servicios -básicamente información militar estratégica- a las potencias europeas (Francia y Gran Bretaña) y americanas (Estados Unidos). De este modo, establecieron contactos con las Fuerzas Armadas francesas, a través del Deuxième Bureau (Inteligencia militar francesa), y se encargaron de vigilar a los nazis en la costa vasca durante la II Guerra Mundial ${ }^{7}$. EI SVI fue un instrumento para la

\footnotetext{
4 Juan PARDO: Crónica de la guerra en el Cantábrico: las fuerzas navales republicanas (1936-1939), San Sebastián: Txertoa, 2004, p. 89. Pedro Barruso: Información, diplomacia y espionaje: la Guerra Civil Española en el Sur de Francia, 1936-1940, San Sebastián: Hiria, 2001, pp. 82 y ss.

${ }^{5}$ Juan Carlos JIMÉNEZ DE ABERÁSTURI y Rafael MORENO: Al servicio del extranjero, op.cit., pp. 41-50. Jon Recondo y Anne Marie Recondo: A los 75 años de Gernika. Un testimonio, Lasarte-Oria: Etor Ostoa, 2011, págs. 325 y ss. Félix Luengo: Espías en la embajada. Los servicios de información secreta republicanos en Francia durante la Guerra Civil, Bilbao: UPV-EHU, 1996, pp. 63-66. Santiago de PABLO: El nacionalismo vasco en la posguerra: Álava 1939-1955, Bilbao: Fundación Sabino Arana, 1991, pp. 25 y ss. Santiago de PABLO: "La resistencia nacionalista en Álava 1936-1955", en Javier Tusell et al. (coord.): La oposición al régimen de Franco. Estado de la cuestión y metodología de investigación, vol. I., Madrid: UNED, 1990, pp. 340 y ss. José Luis de Castro y Alexander UGALDE: La acción exterior del País Vasco (1980-2003), Oñati: IVAP, 2004, pp. 57 y ss. Emilio LÓPEZ ADÁN(Beltza): Nacionalismo vasco en el exilio 1937-1960, San Sebastián: Txertoa, 1977, pp. 14 y ss. José Félix AZURMENDI: Vascos en la Guerra Fría ¿víctimas o cómplices? Gudaris en el juego de los espías, San Sebastián: Ttarttalo, 2013, p. 29 y ss.
}

${ }^{6}$ Laura MANZANERA: Mujeres espías. Intrigas y sabotaje tras las líneas enemigas, Barcelona: Debate, 2008, pp. 234 y ss. M.M. AYUSO et alii: Las mujeres y la guerra civil española, Madrid: Ministerio de Asuntos Sociales, Instituto de la Mujer, Ministerio de Cultura, Dirección de los Archivos estatales, 1991, pp. 251 y ss. Respecto a Juan Ajuriaguerra véase Miguel PELAY: Juan Ajuriaguerra. Su vida, su obra, su muerte, Bilbao: Idatz Ekintza, 1987. Iñaki ANASAGASTI (comp.): Juan Ajuriaguerra en el corazón, Bilbao: Kirikiño, 2008. Cristóbal ROBLES: “En favor de las víctimas de la guerra" , Hispania Sacra LXI, no 124 (2009), pp. 752-753. Santiago de PABLO y Ludger MEES: EI péndulo patriótico. Historia del Partido Nacionalista Vasco (1895-2005), Barcelona: Crítica, 2005, pp. 189.

\footnotetext{
7 Antonio MARQUINA: “El servicio secreto vasco", UNISCI-Discussion Papers, no 10 (2006), pp. 435-449. Alexander UGALDE: "La acción exterior histórica y presente del Gobierno vasco y del Gobierno navarro (1939-2004)", en Joseba AGIRREAZKUENAGA (dir.): Historia de Euskal Herria. Historia general de los vascos, San Sebastián: Lur Argitaletxea, 2004, pp. 259-273. Secundino SERRANO: La última gesta: los republicanos que vencieron a Hitler
} 
consecución de un proyecto político vasco cuyo objetivo era: obtener la colaboración de las potencias europeas antifascistas para combatir a Franco, que -como se sabía- había sido apoyado por Alemania e Italia en la Guerra Civil; mantener activas las instituciones políticas vascas en el exilio, obteniendo a través de sus redes la financiación económica necesaria para su funcionamiento; y recuperar el autogobierno vasco.

En otoño de 1941, en una complicada coyuntura, el presidente vasco llegó a Estados Unidos tras un viaje clandestino de más de un año por la Europa nazi, gracias a las gestiones ante del Departamento de uno de los representantes de la delegación vasca en Nueva York, Manuel Ynchausti ${ }^{8}$. Un periplo que Aguirre completó bajo la identidad falsa del diplomático panameño José Álvarez Lastra, pasando así inadvertido para los nazis. En menos de cuatro años, el gabinete de Gobierno del lehendakari Aguirre se estableció hasta en tres territorios diferentes: Cataluña, Francia y Estados Unidos ${ }^{9}$.

La instalación de Aguirre en Manhattan supuso un punto de inflexión para el eje de la política vasca en el exilio. Por un lado, fijó su atención en mantener preferentemente una estrecha relación con el gigante americano pero, por otro, no renunció a las conexiones establecidas con británicos y franceses. Un viraje que se llevó a cabo en un momento clave: en primer lugar, porque en el verano de aquel año, Estados Unidos y Gran Bretaña firmaron la carta del Atlántico con el objetivo de trabajar conjuntamente en el establecimiento de unas bases de actuación comunes para lograr una paz duradera que respetara el derecho de todos los pueblos a elegir su forma de gobierno (base política sobre la que la directiva vasca podría trabajar en un futuro); y segundo, porque en diciembre, el ejército imperial japonés atacó la base naval de Pearl Harbor, provocando la entrada definitiva de Estados Unidos en la II Guerra Mundial ${ }^{10}$.

Esta última cuestión hizo aún más decisivas las relaciones entre el Gobierno vasco y Estados Unidos. El presidente vasco aprovechó, pues, la tesitura para presentarse como un líder carismático que controlaba una organización política (su gobierno, su partido y el SVI) con amplia implantación en

(1939-1945), Madrid: Aguilar, pp. 224 y ss. Alfonso RINCÓN: Flores de guerra. La odisea de la guerra que no me contaron, Madrid: Ecobook-Editorial del economista, 2010, pp. 484 y ss. Juan Carlos JIMÉNEZ DE ABERÁSTURI y Rafael MORENO, Al servicio del extranjero, op. cit., pp. 117 y ss.

${ }^{8}$ Hacendado vasco-filipino con pasaporte estadounidense muy comprometido con el Gobierno vasco y la causa nacionalista vasca, y amigo personal de José Antonio Aguirre. Véase Jean Claude LARRONDE: Etorri handiko mezenas bat. Manuel Ynchausti, (1900-1961), Villefranque: Instituto Bidasoa, 1998.

9 José Antonio AGUIRRE: De Guernica a Nueva York pasando por Berlín, Madrid: Foca, 2004, pp. 24 y 189 y ss. Koldo San Sebastián: The Basque Archives: Vascos en Estados Unidos (1938-1943), San Sebastián: Txertoa, 1991, pp. 52 y ss. Id., Exilio vasco en América, Vitoria-Gasteiz: Servicio Central de Publicaciones del Gobierno Vasco, 2014. Gloria TOTORICAGÜENA: The Basques of New York. A Cosmpolitan Experience, Vitoria-Gasteiz: Gobierno Vasco, 2004. Carmelo GARITAONAINDIA: José Antonio Aguirre. Primer lehendakari, Oñati: IVAP, 1990, pp. 67 y ss. Ludger MEES: El profeta pragmático. Aguirre: el primer lehendakari (1936-1960), Irún: Alberdania, pp. 69 y ss. Id.: “Aguirre 1960. Atzo 2010 Hoy", Hermes, no 33 (2010), pp. 38-44. Id: “Aguirre, entre carisma y mito", en Ludger MEES y Xosé Manoel NÚÑEZ (coord.): Nacidos para mandar. Liderazgo, política y poder. Perspectivas comparadas, Madrid: Tecnos, 2012, pp. 97-119. Ludger MEES; Santiago de PABLO; José Luis de la GRANJA; José Antonio RODRÍGUEZ RANZ, La política como pasión. El lehendakari José Antonio Aguirre (1904-1960), Madrid: Tecnos, 2014.

${ }^{10}$ Antony BEEVOR: La Segunda Guerra Mundial, Barcelona: Pasado y Presente, 2012, pp. 349 y ss. David M. KENNEDY: Entre el miedo y la libertad. Los EEUU: de la Gran Depresión al fin de la segunda guerra mundial (19291945), Barcelona: Edhasa, pp. 609 y ss. 
América Latina e influyente sobre la opinión pública católica americana ${ }^{11}$. Se presentó como un interesante aliado para las agencias de inteligencia estadounidenses, poniendo en valor el SVI como un útil instrumento para contrarrestar la propaganda fascista en el patio trasero norteamericano ${ }^{12}$.

\section{EL STATE DEPARTMENT Y LAS AGENCIAS DE INTELIGENCIA SE INTERESAN POR LOS SERVICIOS}

Como se ha señalado, Aguirre se asentó en Nueva York gracias a Ynchausti. Pero, también, debido a su mediación y sus lucrativas donaciones Aguirre fue contratado para ocupar un puesto de lectorado en la Universidad de Columbia ${ }^{13}$. Gracias a este puesto, que en aquella época gozaba de gran prestigio, Aguirre pudo acercarse al medio político norteamericano y granjearse unos buenos contactos que tuvieran capacidad de interlocución ante el Departamento de Estado y el Gobierno de Estados Unidos. Carlton Hayes (jefe del Departamento de Historia, amigo personal de Roosevelt y futuro embajador de Estados Unidos en Madrid) fue quien abrió la lata de estas relaciones, pues introdujo al presidente vasco en selectos e importantes círculos políticos, intelectuales y diplomáticos de la sociedad estadounidense, como el del grupo de exiliados católicos europeos que, a posteriori, tuvo un papel protagonista en la reconstrucción de Europa tras la derrota del fascismo ${ }^{14}$. No obstante, las relaciones con los medios políticos oficiales fueron difíciles. Sumner Welles, subsecretario del Departamento de Estado, había mostrado escaso interés ante un posible acercamiento a los nacionalistas vascos, a quienes consideraba un grupo minoritario de escasa influencia, cuya relación con el Gobierno estadounidense podía irritar a Franco. Tampoco la Casa Blanca se mostró favorable a que el presidente Roosevelt se relacionara con el grupo liderado por Aguirre, pues le consideraron -al igual que Wellesun líder político de un grupo minoritario y poco representativo que no mantenía relación ni con el Gobierno de Franco ni con los políticos republicanos conocidos en Washington ${ }^{15}$.

En esta tesitura, el lehendakari-inundado de optimismo- dio un nuevo impulso a su estrategia. Dirigió su atención hacia el sector católico de la Administración Roosevelt, encabezado por el vicepresidente de Estados Unidos Henry A. Wallace, un político del Partido Demócrata, intensamente

${ }^{11}$ Ludger MEES: El profeta pragmático, op. cit., pp. 43 y ss.

${ }^{12}$ Centro de Patrimonio Documental de Euskadi-Irargi, “Informe no 132. 2a visita a Mr. Wendelein en el ministerio de Estado", sin lugar, 15-2-1939, Fondo GE-0041-05.

13 Columbia University Archives (CUA), "Nomination for appointment", Nueva York, 23-9-1941, Historical Biographical Files Collection, Box 77, Folder Aguirre. En esta nominación se especifica que el salario anual de Aguirre será de 4.500 dólares por donativo especial realizado por Ynchausti. Véase al respecto Iñaki GOIOGANA: "José Antonio Aguirre, profesor de la Columbia University", en José Antonio ASCUNCE ET AL.: Exilio y Universidad (1936-1955), San Sebastián: Saturraran, 2008, pp. 599-643. Gloria TOTORICAGÜENA: “José Antonio de Aguirre y Lecube in New York", Euskomedia (2004), en http://www.euskonews.com/0245zbk/kosmo24501.html , consultado el 22-05-2016). El 10 de octubre, la Universidad de Columbia hizo oficial su contratación a través de una nota de prensa de la Associated Press recogida en CUA, "Ex President of Basques will teach at Columbia", Montevideo, 10-10-1941, Asociated Press, Columbia University Archives, Historical Biographical Collection, Box 1, Folder Aguirre.

${ }^{14}$ Entre los exiliados católicos europeos sobresalen el italiano Luigi Sturzo (fundador del Partido Popular italiano), Frans van Cauwelart (expresidente del Parlamento belga) y Jacques Maritain (filósofo, profesor en la Columbia University). Ludger MEES(et al.): La política como pasión, op. cit., Tecnos: Madrid, 2014, p. 446.

${ }^{15}$ Juan Carlos JIMÉNEZ DE ABERÁSTURI y Rafael MORENO: Al servicio del extranjero, op.cit., pp. 403-404. 
católico y propenso a promover la causa vasca dentro del Gobierno de Estados Unidos por razones religiosas y morales. Wallace era un hombre muy popular, pero pertenecía al ala más izquierdista del Partido Demócrata, siendo criticado tanto dentro como fuera de su partido por ser presuntamente cercano al comunismo ${ }^{16}$. Aun así, a la altura de 1942, contaba con gran apoyo popular y estaba en la cima de su carrera política, aunque pronto entrara en declive. El vicepresidente, que era un político muy interesado por los asuntos hispanoamericanos, se sintió atraído por las ideas que expuso Aguirre en un congreso organizado por el exilio republicano español en Estados Unidos. A partir de entonces establecieron un diálogo regular y se inició así una relación bastante estrecha, que no sólo provocó recelos y críticas en el Gobierno estadounidense sino también en el Partido Demócrata ${ }^{17}$.

En efecto, el buen entendimiento del tándem Wallace-Aguirre levantó ampollas dentro de algunos círculos de poder estadounidenses que, diligentemente, se preocuparon por erosionar los vínculos de esta asociación. Un complicado contexto que llevó al lehendakari y a Ynchausti a explorar otras opciones. Por un lado, la Coordinator of Inter-American Affairs (CIAA) de Nelson Rockefeller, una agencia estadounidense encargada de velar por la seguridad del hemisferio occidental. Por otro, la Coordinator Office of Information (COI), una agencia de inteligencia y propaganda creada por Roosevelt en julio de 1941 y dirigida por el coronel William J. Donovan ${ }^{18}$. Los contactos con la agencia de Rockefeller sirvieron para que Ynchausti y Aguirre avanzaran en su objetivo de establecer un acuerdo de colaboración con Estados Unidos para luchar contra el Eje en Sudamérica. Según ha señalado Juan Carlos Jiménez de Aberásturi, la organización vasca presentó un proyecto de colaboración en el que ponían a su disposición todos sus recursos en Sudamérica para emprender actividades de propaganda y espionaje. Para ello necesitaban una financiación inicial de 30.000 dólares y una dotación presupuestaria que permitiera a Euzko Deya, el periódico oficial del Gobierno vasco que se editaba en Buenos Aires, duplicar su tirada ${ }^{19}$.

Sin embargo, las negociaciones se pospusieron, porque las propuestas de colaboración vasca generaron controversias en algunos sectores del Gobierno estadounidense, que consideraban contraproducente encargar esta labor propagandística e informativa a un grupo del exilio republicano español, cuando aún había facciones de este último que mantenían rencillas y diferencias políticas en América ${ }^{20}$.

Tras superar diferentes obstáculos, las gestiones salieron adelante debido a la irrupción del Servicio de Inteligencia británico en la escena negociadora. Los británicos conocían la potencialidad de los Servicios Vascos desde el inicio de la guerra, ya que habían colaborado con ellos en Londres y Nueva York y estaban al tanto de su eficacia. Así, a partir de diciembre de 1941 los británicos estrecharon su colaboración con los vascos, al haber obtenido las claves e instrucciones secretas que la Marina

\footnotetext{
16 John Maze GRAHAM: Henry A. Wallace: his Search for a New World Order, University of North Carolina Press: Chapel Hill, 2009.

${ }^{17}$ Juan Carlos JIMÉNEZ DE ABERÁSTURI: De la derrota a la esperanza: políticas vascas durante la Segunda Guerra Mundial (1937-1947), Oñati: IVAP, 1999, p. 476.

${ }^{18}$ Eugene LIPTAK: Office of Strategic Services 1942-45. The World War II Origins of the CIA, Osprey: New York, 2009, pp. 4-5.

19 Juan Carlos JIMÉNEZ DE ABERÁSTURI: De la derrota a la esperanza, op. cit., pp. 476.

${ }^{20}$ Ludger MEES(et al.): La política como pasión, op.cit., p. 450.
} 
franquista había entregado a los buques españoles, en caso de que España entrara en la II Guerra Mundial. Esta relación se centró en la grabación por parte del lehendakari de un mensaje radiofónico en castellano y en euskera que daba instrucciones a los marineros vascos de los buques españoles para que actuaran a favor de los Aliados en el hipotético caso de que España entrara en la contienda. Este tipo de actividades de colaboración con la causa aliada llevaron a los diplomáticos británicos a adoptar la siguiente actitud con respecto a los vascos: aprobar todo tipo de iniciativas que evitaran la entrada de Franco en la guerra, obteniendo importantes beneficios de una colaboración que políticamente no les comprometía a nada. Las diferentes agencias estadounidenses que siguieron de cerca esta política constataron que también podían beneficiarse de estos servicios, desoyendo a aquellos sectores del Departamento de Estado contrarios a la cooperación. De este modo, colaboraron con las organizaciones de Inteligencia británica en materia de contraespionaje e informaron al presidente Roosevelt de las actividades vascas sin tener un contacto formal con ellos.

En noviembre de 1941, William Stephenson, principal cabeza visible de la British Security Coordination (BSC) y director del Secret Intelligence Service (SIS), servicio de inteligencia especial adscrito a la BSC, contactó con Aguirre, para tratar los puntos de un plan de colaboración en labores informativas para América. Sin embargo, con la entrada de Estados Unidos en la guerra, estas gestiones quedaron en un segundo plano. De hecho, se solaparon con los contactos establecidos con el COI, un servicio para la recogida de información secreta en el extranjero que había sido creado en diciembre de 1940 por el general William Donovan, siguiendo el encargo de Franklin D. Roosevelt de centralizar los servicios de inteligencia estadounidense en una única organización y dirigir en exclusiva las actividades de información en Latinoamérica. Esta centralización, que progresivamente apartó al BSC del escenario latinoamericano, conllevó la readecuación de las estructuras organizativas de las agencias de información y la creación de nuevos puestos de responsabilidad que abrieran de nuevo las puertas a una colaboración más estrecha con el Gobierno de Estados Unidos.

De este modo, el 5 de mayo de 1942, el lehendakari Aguirre se reunió con Allen Dulles, coordinador del $\mathrm{COI}$ en Nueva York y con Gregory Thomas, director de esta misma institución en España ${ }^{21}$. Durante la entrevista, el presidente vasco les explicó que antes de su llegada a Estados Unidos había recorrido varios países de Sudamérica, en los que había podido comprobar la existencia de un importante sector social amante de la libertad (en sentido demócrata-cristiano), que tenía como modelo a seguir la democracia estadounidense y que, en su opinión, debía ser explotado en beneficio de la política de Buena Vecindad de Roosevelt. El mejor procedimiento para promover un acercamiento de estos estratos sociales a la política del Gobierno de Estados Unidos, añadió el lehendakari, era el empleo de clérigos vascos residentes en Latinoamérica que, por su fe en la democracia y su ideología cercana al nacionalismo vasco, podían ayudar desde el púlpito a la expansión del mensaje de libertad y lucha contra el totalitarismo europeo. Allí, además, había una gran masa de población de origen vasco que había emigrado durante el siglo XIX; las personalidades de ascendencia vasca gozaban de gran reputación, al haber dirigido Gobiernos en Argentina como era el caso de José Félix Uriburu (1930-1932) y Roberto Ortiz (1938-1942), y en Uruguay, en el de Juan Campisteguy (1927-1931). Asimismo, en estos y otros países, como Colombia y Chile, había una amplia red de centros vascos, juntas extraterritoriales de partido (PNV fundamentalmente) y asociaciones de orientación nacional vasca que podían ser

\footnotetext{
${ }^{21}$ Richard H. SMITH: OSS: The Secret History of America's First Central Intelligence Agency, First Lyon Press: Guilford, 2005, p. 73.
} 
utilizadas por la acción exterior del Gobierno vasco en el exilio y por los Servicios ${ }^{22}$. Por tanto, teniendo en cuenta estas cuestiones, Aguirre les informó de que había mantenido contactos con Wallace, con quien había hablado sobre la posibilidad de establecer un acuerdo de colaboración entre el Servicio Vasco de Información -muy bien asentado y organizado en Sudamérica-y las agencias estadounidenses (principalmente el COI) para contrarrestar la propaganda totalitaria del $\mathrm{Eje}^{23}$.

En este contexto, el 15 de mayo de 1942, Manuel de la Sota presentó al COI, la agencia de Inteligencia estadounidense, un plan de cooperación para combatir a los grupos afines al nazismo y al falangismo en Latinoamérica mediante la propaganda, el control de las fuentes información y la utilización del $\mathrm{SVI}^{24}$. Para garantizar el buen funcionamiento de todo este entramado, los jefes de la organización vasca organizarían y coordinarían las actividades de este proyecto y contarían con libertad de movimientos y la documentación correspondiente, medios seguros y confidenciales de transmisión de información y aparatos de radio con códigos de cifrado estadounidenses.

Tras la presentación de esta propuesta, el 22 de mayo Aguirre recibió la noticia de que la colaboración con los norteamericanos debía empezar de inmediato, pues ya habían llegado a un acuerdo en materia económica por el que recibirían periódicamente financiación para cubrir este tipo de labores en Latinoamérica. Probablemente también para ayudar en el interior de España y en la Francia ocupada, y organizar desde dentro redes de colaboración con los Aliados ${ }^{25}$. Con toda seguridad, tal y como han indicado los principales expertos en el tema, el acuerdo definitivo de cooperación entre la organización vasca y la estadounidense se firmó en algún momento de mayo de 1942. Sin embargo, se desconoce la fecha, la firma y el contenido exacto de ese documento, sin duda el más importante de la relación entre el Gobierno vasco y Estados Unidos ${ }^{26}$. Ahora bien, es muy posible que el texto del acuerdo oficial recogiera en gran parte el plan presentado por Manuel de la Sota el 15 de mayo ${ }^{27}$. Es decir, que el Gobierno vasco aportara ayuda a la propaganda pro aliada, información sobre círculos favorables al Eje y servicios de espionaje, especialmente en Latinoamérica, a cambio de dinero, el mantenimiento de unas estrechas relaciones políticas y otras cuestiones menores (como el uso de valija diplomática por parte de los representantes vascos). En cualquier caso, apenas un mes más tarde, lo que Aguirre denominó "Comité Donoban" se transformó en la Office of Strategic Services (OSS), "una

\footnotetext{
${ }^{22}$ Sobre la implantación vasca en América, véase Óscar ÁLVAREZ GILA (et al.): Delegaciones de Euskadi (19361975), op. cit. José Manuel AZCONA: El dogma nacionalista vasco y su difusión en América (1890-1960). Un paradigma de paradiplomacia, Gijón: Trea, 2015.

${ }^{23}$ National Archives and Records Administration (NARA), "Memorándum de conversación sobre José Antonio Aguirre, en el que participan Gregory Thomas y Allen W. Dulles, enviado por Spencer Phenix a Hugh Wilson", Washington DC, 6-5-1942, RG 226, OSS Files, Entry 106, Box 32, Folder 151.

${ }^{24}$ NARA, "Plan for the coordination of Basque Services submitted by Manuel de la Sota on behalf of President José Antonio Aguirre of the Basque Republic", Washington DC, 15-5-1942, NARA, RG 226, OSS Files, Entry 106, Box 32, Folder 151.

${ }^{25}$ Juan Carlos JIMÉNEZ DE ABERÁSTURI: De la derrota a la esperanza, op.cit., p. 477.

${ }^{26}$ A pesar de haber intentado localizarlo, nuestras pesquisas en los NARA no dieron el resultado esperado. Sin embargo, tal y como indican los autores de La política como pasión (p. 452), "Xabier Irujo resume el contenido de este acuerdo, pero no señala ninguna fuente". Véase Xabier IRUJO: Expelled from the Motherland. The Government of the President Jose Antonio Aguirre in Exile (1937-1960), Reno: University of Nevada, 2012, pp. 122 y ss.

${ }^{27}$ Xabier IRUJO, Expelled from the Motherland, op.cit., p. 124.
} 
importante agencia de espionaje (...), antecesora de la Central Intelligence Agency (CIA), fundada en $1947^{\prime 28}$.

Aunque hubo funcionarios del Departamento de Estado como Joseph J. Montllor (CIAA), Lawrence Duggan y Selden Chapin (Division of American Republics) que vieron a los vascos como un útil instrumento de propaganda aliada en Latinoamérica, fue William J. Donovan quien confió con más decisión en la organización vasca, como se demuestra con la gira propagandística que realizó el lehendakari por América Latina y la colaboración de los Servicios vascos con el espionaje norteamericano en Latinoamérica y Europa ${ }^{29}$.

Bajo control de los estadounidenses, la red vasca pronto se profesionalizó. Los agentes vascos se encargaron de identificar a los miembros de las tripulaciones y los pasajeros sospechosos de navíos procedentes de Europa, de informar sobre movimientos desacostumbrados del barco y actividades de los oficiales de la policía secreta española, y de obtener pruebas del espionaje alemán y datos de la situación política, social y económica en España. En 1943 los servicios de espionaje aliados ya contaban con varias personas de confianza en todos los barcos españoles que cruzaban el Atlántico. Se encargaron, pues, de vigilar a buques que tuvieran por destino Estados Unidos y Argentina, como ocurrió con el Cabo de Buena Esperanza y el Cabo de Hornos que fueron dos auténticos nidos de espías nazis $^{30}$. En estas labores, la organización vasca cobró especial importancia por su red de información, extendida por España y Francia y con conexiones en América. Así lo señaló Gregory Thomas a William Donovan en abril de 1943:

Although profoundly Catholic, the Basques in the Spanish Civil War opposed General Franco and the members of the Basque Government led by President Aguirre are now living in exile. Aguirre is in the United States and has maintained the closest cooperation with OSS for the purpose of affording the intelligence facilities of his people to the United States Government in the present war. [...] the majority of the Basque people and their political leaders have maintained an intelligence network in Spain which stretches into France, penetrating some of the highest German military circles as far north as Paris. Inside Spain the Basque intelligence operatives who have remained following the Spanish Civil War maintain communications through members of this organization who work as personnel of ships between Spain, South and Central America and the United States. Much information of a military as well as a political nature is made available to the Basques in exile through Basque priests who travel from Spain to South America, there are no Spanish ships which do not have Basque personne ${ }^{31}$.

Los observadores infiltrados en estas naves fueron desde el capellán del barco, como el caso de Luis Petralanda (naviera Ybarra), al capitán, como sucedió con Antonio Camiruaga (naviera Ybarra), pasando por un importante número de agentes profesionales, como José Laredogoitia Menchaca que decidieron arriesgar su vida para defender los intereses de los Aliados. El caso de este último es singular

\footnotetext{
${ }^{28}$ Ludger MEES (et al.): La política como pasión, op.cit., p. 452.

${ }^{29}$ NARA, "Carta de William J. Donovan a Cordell Hull", Washington DC, 3-7-1942, RG 59, State Department, Box 5234, Leg. 852.01/707.

${ }^{30}$ Mikel RODRÍGUEZ: Espías Vascos, op. cit., p. 160 y ss. Iñaki BERNARDO e Iñaki GOIOGANA: Galíndez: la tumba abierta Guerra, exilio y frustración, Bilbao: Fundación Sabino Arana, 2006, , p. 278.

${ }^{31}$ NARA, "Memorándum de Gregory Thomas para William J. Donovan", sin lugar, 9-4-1943, NARA, RG. 226, OSS Files, Entry 106, Box 32, Folder 151.
} 
por tratarse de un agente doble que trabajó en la oficina de la Abwehr -Inteligencia Militar Alemanade Bilbao para evitar ser encarcelado, posiblemente tras ser acusado de nacionalista vasco por las autoridades españolas. Pero pronto cambió de bando. Durante una de sus primeras misiones en Brasil, comenzó a colaborar con el Servicio Vasco de Información, manteniéndose como infiltrado dentro de la organización alemana y obteniendo importantes datos que permitieron el desmantelamiento de varias células de espionaje y propaganda nazi en Sudamérica ${ }^{32}$.

A pesar de estos éxitos, la pugna por el control de los operativos protagonizada por las agencias militares y civiles estadounidenses afectó a la eficiencia del SVI. Desde principios de 1942, el Joint Chiefs of Staff (JCS) o Junta de jefes del Estado Mayor, y el Departamento de Guerra se habían propuesto eliminar al $\mathrm{COI}$-a su modo de ver, principal coartador de su libertad de actuación- para desmembrarlo en diferentes agencias civiles y militares. En realidad, su objetivo era limitar el control del grupo de Donovan sobre las operaciones de espionaje y socavar los contactos clandestinos de esta organización en Europa y Sudamérica. Finalmente, los jefes del Estado Mayor Conjunto, que ansiaban controlar los proyectos y misiones de inteligencia, lograron que todas las decisiones fueran consensuadas con el Departamento de Estado y que las agencias dedicadas a labores de espionaje se dividieran en civiles y militares. Por este motivo desapareció el COI, como coordinador de todas las actividades de inteligencia, y nació la OSS, la Oficina de Servicios Estratégicos -antecedente de la CIA-, para supervisar y analizar la información del Estado Mayor Conjunto y llevar a cabo operaciones no previstas por ninguna otra agencia. El nuevo organismo pronto se vio limitado por varios flancos, por un lado, el FBI, con exclusividad jurisdiccional de actuación en Latinoamérica, y por otro, el Ejército -concretamente la sección G-2 dirigida por el General Strong- con ámbito de actuación prácticamente total, debido al estado de situación bélica ${ }^{33}$

En los planes de Donovan para España y Latinoamérica, el Servicio Vasco de Información tuvo un importante papel como organización de espionaje independiente. En el caso de la Península la colaboración vasca podía ser fundamental para los operativos de la OSS, ya que podían ayudarles a cruzar la frontera con Francia de forma clandestina y además, en caso de que Hitler decidiera ocupar España, contar con ellos para luchar contra los alemanes. Donovan consiguió así establecer una efectiva colaboración con los vascos que tuvo su momento más álgido durante la II Guerra Mundial y que no estuvo exenta de dificultades por los problemas jurisdiccionales con el $\mathrm{FBI}$, la OSS y el ejército estadounidense y por el intento de control de todas las agencias de las actividades del SVI. Para evitar problemas de funcionamiento, la dirección del SVI fue pragmática y decidió colaborar con todas las organizaciones por "el bien de la democracia". Sin embargo, esa colaboración conllevaba una contraprestación no sólo económica (ya mencionada), sino también política; es decir, que las agencias estadounidenses con las que estaban cooperando tenían que ayudar a derrocar al franquismo, recuperar la democracia en España y el autogobierno vasco.

\footnotetext{
${ }^{32}$ Las labores de espionaje de José Laredogoitia Menchaca se recogen ampliamente en David MOTA ZURDO: “José Laredogoitia Menchaca, el agente Gernika", Sancho el Sabio no 37 (2014), pp. 159-179. Véase también Rafael MORENO IZQUIERDO: "Bromo, el ovejero que espió para todos", El País, 2-11-2014, recurso electrónico consultado el 15-05-2016: http://cultura.elpais.com/cultura/2014/10/31/actualidad/1414769173 391482.html.

${ }^{33}$ Bradley F. SMITH: The Shadow Warriors: OSS and the Origins of the CIA, New York: Basic Books, 1983, pp. 117119.
} 


\section{ESPIANDO A NAZIS Y COMUNISTAS}

A finales de diciembre de 1943, José María Lasarte se puso en contacto con Antón Irala para transmitirle información relacionada con la actividad de los Servicios en el interior de España, la cual había sido conseguida a través de una red de marineros vascos que transportaban correspondencia clandestina dentro de los buques españoles que hacían ruta entre Europa y América. Una carta escrita a finales de octubre por el líder del PNV en el interior, Juan Ajuriaguerra, puso de manifiesto el disgusto del servicio secreto vasco que operaba en la Península Ibérica con la OSS, a la que acusaba de descoordinación y desatención de los proyectos de ampliación de las labores de espionaje en el sur de Francia $^{34}$.

Tratando de clarificar el funcionamiento de los Servicios, las autoridades vascas decidieron trabajar para la agencia de Hoover en Sudamérica y para la OSS y el ejército estadounidense en Europa. Así, Antón Irala se entrevistó con Jerome Doyle, uno de los agentes del Special Intelligence Service (SIS) del FBI, para emprender las gestiones conducentes al establecimiento de una colaboración exclusiva en América $^{35}$. Los deseos del lehendakari Aguirre y de Irala eran limitar los contactos en el hemisferio occidental a una única organización, pero para que así fuera, esta debía asumir la financiación de las actividades vascas sobre el terreno. Además, según lo hablado en esta entrevista, tratarían solo con los Legal Attachè -agregados legales del FBI en las embajadas de Estados Unidos en Sudamérica-, a quienes los agentes vascos acudirían para recibir información sobre las misiones e instrucciones ${ }^{36}$. La financiación de estas operaciones se tasó en 4.500 dólares mensuales, fondos que debían ser enviados a Aguirre para que se encargara de distribuirlos porque "the Basques will perform for more ably as they would then be working for patriotic motives and not as paid agents of the United States Government" ${ }^{37}$. De este modo, el SVI se presentó también como un instrumento contra la penetración del comunismo en Latinoamérica, que contaba con agentes dentro de los partidos políticos de este signo en Colombia, México, Cuba, Argentina y Chile, tal como explicaba el agente especial del FBI C. H. Carson:

\footnotetext{
${ }^{34}$ NARA, “Memorándum de E.W. Andrews a Frank T. Ryan sobre la relación con agentes vascos”, sin lugar, 3-21944, RG 226, OSS Files, Entry 210, Box 487, WN 18910, Leg. 7024 X.

35 Juan Carlos JIMÉNEZ DE ABERÁSTURI: De la derrota a la esperanza, op. cit., p. 478.

${ }^{36}$ El programa de Legal Attaché del FBI nació dentro de la vanguardia de la política de Buena Vecindad de Roosevelt para combatir las operaciones alemanas y, posteriormente comunistas, en Sudamérica. Los agregados legales formaban parte de la plantilla de la embajada de Estados Unidos, su misión era mantener informado al embajador sobre las actividades del FBI y establecer buenas relaciones con las autoridades locales y otras agencias de inteligencia establecidas en la zona, en I. C. SMITH: Inside. A Top G-Man Exposes Spies, Lies, and Bureaucratic Bungling Inside the FBI, Nashville: Thomas Nelson Press, 2009, p. 103. Tim WEINER, Enemigos, Una Historia del FBI, Barcelona: Debate, 2012, pp. 161-162. Athan G. THEODARIS: The FBI: A Comprehensive Reference Guide, Phoenix: The Orix Press, 1999, p. 338.

37 FBI Archive, Memorándum “Latin-American Matters” de E.E. Comroy, SAC (Special Agent in Charge), a J.E. Hoover, Nueva York, 4-3-1944, Basque Intelligence Service Section II, NND 267.468. FBI Archive, Memorándum "Basque Organization. Latin American Matters" enviado por E.E. Conroy a J.E. Hoover, Nueva York, 18-4-1944, Basque Intelligence Service Section II, NND 267.468. En cuanto a la cifra Mikel Rodríguez señala 6.500 dólares al mes, véase Mikel RODRÍGUEZ: Espías vascos, op. cit., p. 212.
} 
Our experience with the Basques in Latin America has indicated that they are extremely valuable informants, and they have been of assistance to the Legal Attaches in a number of countries. [...]. In view of their interest in combating Communism and their efforts already begun to infiltrate Communist organizations, they could be of extreme value in the future, inasmuch as Communism is presently one of the principal problems in Latin America, and will became increasingly serious ${ }^{38}$.

Los diferentes Legal Attachè del FBI llegaron a un acuerdo con los jefes locales de los Servicios vascos, por el que se comprometieron a colaborar en labores de información sobre actividades comunistas en Sudamérica, poniendo un énfasis especial $-y$ en el punto de mira- al exilio político español. Asimismo, Lasarte recibió la orden de colaborar con el FBI para crear una red de información en Argentina que se encargara de infiltrar agentes en los círculos políticos, económicos y religiosos, con vistas a una posible ampliación hacia Bolivia, Uruguay, Paraguay y Chile. En apenas unos meses, la organización vasca amplió sus labores de información para el FBI, investigando las actividades comunistas en estos países, junto con el control de los movimientos y operaciones de los nazis y el examen de los pasajeros y miembros de las tripulaciones de los buques españoles que ya venía realizando. Antón Irala ya había advertido reiteradamente a los estadounidenses que en un futuro cercano el comunismo se convertiría en su principal problema. En un viaje que realizó Irala en 1943 por varias repúblicas sudamericanas había comprobado in situ que no solo los simpatizantes comunistas eran más numerosos que los falangistas y fascistas, sino que además se preparaban para llevar a cabo una auténtica revolución social que acabara con la influencia estadounidense en este territorio. Así lo explicó en un encuentro con agentes del FBI: "both such movements, namely, Communist and Fascist, have for their inmediate aim the destruction of North American influence in Latin America and the ultimate launching of revolutionary governments along the Soviet lines" ${ }^{39}$.

Para mediados de 1944, la organización vasca ya estaba operativa en los principales países de habla hispana de norte, centro y sur de América. En México consiguió introducirse dentro de algunas organizaciones comunistas y elaborar listas de sospechosos españoles, italianos, alemanes y algunos nativos simpatizantes del Eje. En Santo Domingo, Jesús Galíndez -delegado del Gobierno vasco en la República Dominicana- logró una notable influencia sobre el gobierno del dictador Rafael Leonidas Trujillo, así como acceso a los principales círculos sociales del país, llevando a cabo diferentes misiones para las agencias de información estadounidenses, pese a contar con escasos colaboradores ${ }^{40}$. En Cuba, las personalidades de origen vasco que estaban bien situadas en la vida pública, junto con un importante grupo de refugiados de la Guerra Civil española, trabajaron de forma clandestina, informando sobre las actividades del Centro de independientes, Centro Gallego, Centro Catalán, el Círculo Republicano Español y el grupo comunista de Ernest Hemingway ${ }^{41}$. Sin embargo, una vez más, la

\footnotetext{
${ }^{38}$ FBI Archive, Memorándum para el Sr. Ladd "Basque organization-Latin American Matters”, Nueva York, 14-31944, Basque Intelligence Service, Section II.

${ }^{39}$ FBI Archive, "Carta enviada de un informante confidencial a J.E. Hoover", Cuba, 24-4-1944, Basque Intelligence Service, Section II.

${ }^{40}$ Según se ha señalado en varias investigaciones Galíndez trabajó tanto para el agregado militar de la embajada de Estados Unidos en la República Dominicana como para el agregado legal del FBI. De hecho, en el FBI recibió un nombre en clave, agente Rojas (NY 507-S), agencia para la que trabajaría hasta 1956, fecha de su desaparición. Juan Carlos JIMÉNEZ DE ABERÁSTURI: De la derrota a la esperanza, op. cit., p. 483.

${ }^{41}$ Las últimas investigaciones han señalado que Ernest Hemingway fue contratado por el agregado legal del FBI en la embajada de Estados Unidos en la Habana, para dirigir una red de espías que siguiera los pasos a posibles
} 
escasez de recursos y la falta de directrices concretas obstaculizaron la continuidad de estas operaciones.

En el verano de 1943 el espionaje alemán dejó de ser la preocupación exclusiva de Estados Unidos. Se habían desvanecido los temores a sufrir una posible invasión por las fuerzas del Eje, pero rápidamente había surgido otra amenaza: el comunismo, que -según empezaban a valorar instituciones como el FBI- pretendía socavar la imagen de salvadores de la democracia que la Casa Blanca se había preocupado por construir ${ }^{42}$. Para evitarlo, Hoover comenzó a promover un lobby político en Washington que convenciera a la Administración Roosevelt de que o se paraban los pies a Stalin o el ejército rojo continuaría avanzando hacia Occidente, ganando adeptos y debilitando a las democracias $^{43}$. Tal era el caso de Sudamérica, en donde -según Hoover- debían actuar rápidamente, sobre todo en México, pues la influencia de los comunistas se estaba haciendo cada vez más notoria, como se dedujo de los informes del Servicio Vasco de Información.

En mayo de 1944, Antón Irala y el agregado legal del FBI en México se reunieron para tratar en detalle la colaboración del SVI con dicha agencia. El agente estadounidense le solicitó una lista de 300 hombres vascos con sus direcciones, ocupaciones e información especial sobre su pasado, que vivieran en México D.F. y otras ciudades de importancia, para ser utilizados como fuentes de información. Irala designó de entre todos a cinco hombres para que se dedicaran -bajo su supervisión- a labores de investigación. Los norteamericanos le solicitaron un historial completo, que incluyera las actividades realizadas en los últimos cuatro años, que mostrara su nivel de inglés, su estado civil (preferente: soltero) y su disposición a trabajar en la estructura de la inteligencia vasca que se pondría a disposición del FBI. Este grupo trabajaría de forma temporal -no más de cinco meses-, recibiendo por ello un sueldo de unos 300 pesos mensuales. Además, la agencia norteamericana se reservó la posibilidad de anular el acuerdo en cualquier momento si consideraba insatisfactorias las labores de los vascos. Estas exigencias llevaron al dirigente vasco a transmitir su descontento al FBI. Para Irala el salario era muy bajo, menos de lo que la mayoría ganaba en sus negocios privados. Y es que, en la mayoría de los casos, en función de la misión y el objetivo, la inversión superaba los 1.000 pesos semanales ${ }^{44}$.

quintacolumnistas españoles -falangistas principalmente-. Sin embargo, en 1940 escribió un manifiesto contra el $\mathrm{FBI}$ que, junto sus actividades a favor de la II República durante la Guerra Civil española, le colocaron rápidamente en el punto de mira de Hoover, llegándose a afirmar que era uno de los miembros del Partido Comunista de Estados Unidos. Tampoco ayudó a esto que durante su estancia en China pasara información sobre el Gobierno de su país a Harry Dexter White que, sin quererlo, acabó en el Kremlin por las vinculaciones de este último con el espionaje soviético. Véase Peter MOREIRA: “Ernest Hemingway, agente secreto", Arcadia, no 7 (2006), p. 10 y ss. Id., Hemingway on the China Front: His WWII Spy Mission with Martha Gellhorn, Dulles: Potomac Books, 2006. Edorta JIMÉNEZ: San Fermingway. Otras historias de Ernest Hemingway, Tafalla: Txalaparta, 2005, p. 195 y ss.

42 Algunas investigaciones señalan que el ejército estadounidense se preocupó por vigilar muy de cerca las actividades alemanas en Latinoamérica por miedo a una posible intervención de algún tipo de red clandestina en Estados Unidos. Max P. FRIEDMAN: Nazis and Good Neighbors: The United States Campaign Against the Germans of Latin America in World War II, Cambridge: Cambridge University Press, 2003, pp. 159 y ss. Glenn YEADON y John HAWKINS: The Nazi Hydra in America: Suppressed History of a Century, Joshua Tree: Progressive Press, 2008, p. 47 y ss. y 163-191.

${ }^{43}$ Tim WEINER, Enemigos, op. cit., p. 163.

${ }^{44}$ Otros datos indican que el Servicio Vasco de Información costaba al FBI 4.600 dólares mensuales. Juan Carlos JIMÉNEZ DE ABERASTURI: De la derrota a la esperanza, op. cit., p. 478. 
Tras consultar con el lehendakari los términos de esta conversación, se reunió de nuevo con Doyle a mediados de junio para señalarle que habían tomado la decisión de restar importancia a la cuestión de los pagos pues, ante su deseo de colaborar con el FBI, estaban dispuestos a pagar la diferencia entre la cantidad que les ofrecían y la exigida por los agentes vascos; incluso, si fuera necesario, a llevar a cabo estas labores sin contraprestación económica ${ }^{45}$. Aunque durante un tiempo trabajaron sin problema, las fricciones reaparecieron en octubre-noviembre de 1944, cuando el FBI, de forma unilateral, decidió reducir la financiación de los Servicios, aduciendo bajo rendimiento. Tal actitud merecía a Irala esta opinión:

Sin mengua del sincero aprecio personal, y de la estimación verdadera de su Causa, que es la nuestra, entiendo que hay de parte de ellos una falta de confianza y de comprensión hacia nosotros. A mi modo de ver nada justifica la medida de reducción aunque como es natural, no pretenda haber alcanzado la perfección y no disimule nuestros fallos y hasta nuestros fracasos parciales. No me explico cómo en las circunstancias presentes, se puede decir que nuestro trabajo no vale el importe de su asignación cuando a nosotros trabajando austeramente nos cuesta bastante más. [...] el aspecto económico no puede preocupar a los amigos para quienes en el volumen de su presupuesto tengo la seguridad de que es una verdadera insignificancia. Tal vez, les resulta difícil, por hábitos profesionales, habituarse a tratar, en vez de con agentes, con un servicio modesto, todo lo modesto que se quiera, pero que no es un grupo de individuos agentes suyos, sino el SI [Servicio de Inteligencia] de una organización patriótica que trabaja creyendo servir a la Causa común de la libertad, y a la vez a su causa patriótica. Mientras no haya la sincera comprensión de este principio, no solo intelectual sino prácticamente, las cosas no andarán bien. Por mi parte, mi conciencia me impide trabajar sino es a base de este principio. Si el decir esto, es innecesario, por inmerecido, atribúyase a que lo sucedido ha causado en mí un desencanto y un principio de depresión que creo obligado no ocultar ${ }^{46}$.

Hoy sabemos que Edgar Hoover sospechó del Servicio Vasco de Información, como de cualquier otra organización que tuviera algún tipo de compromiso con la OSS de Donovan, muy interesada en trabajar estrechamente con el espionaje soviético en Europa para acabar con Hitler. Hoover creía que la OSS estaba infestada de agentes de Stalin que solo buscaban obtener información sobre la situación interna de las agencias de Estados Unidos ${ }^{47}$.

En consecuencia, en noviembre de 1944 Antón Irala renunció a sus funciones como coordinador de los servicios de inteligencia vascos en Latinoamérica, dejándolos en manos de José María Lasarte y Manuel de la Sota. Se incorporó así a una misión de la OSS en Francia que se inició después del desembarco de Normandía para organizar una red de información entre los Pirineos, Francia y Bélgica. Y es que, al otro lado del Atlántico, casi un año después de ser expresadas, las quejas de Ajuriaguerra surtieron efecto y, a finales de agosto de 1944, la OSS emprendió un nuevo proyecto de colaboración con los vascos en el sur de Europa. Los objetivos de Ajuriaguerra eran la preparación de los trabajos preliminares para el desarrollo de un servicio secreto vasco de información en Francia y Bélgica, la ampliación de este servicio con varias de sus células en España, la adquisición de información en Sudamérica, independientemente de los canales ya existentes con el FBI en asuntos relacionados con la

\footnotetext{
${ }^{45}$ FBI Archive, "Carta de E.E. Conroy a J.E. Hoover", Nueva York, 23-6-1944, Basque Intelligence Service Section II.

${ }^{46}$ FBI Archive, “Carta de Antón Irala a José Antonio Aguirre”, Nueva York, 14-11-1944, Basque Intelligence Service Section VII, NND-267468, Leg. 64-23117.

${ }^{47}$ Tim WEINER, Enemigos, op. cit., p. 171.
} 
Hispanidad, y la creación de una red de correo clandestina dirigida por Gregory Thomas (alias Argus) en España y Francia que enviara documentación a Washington a través de Lisboa y París ${ }^{48}$. El Departamento de Estado no apoyó este proyecto, como se deduce de un memorándum enviado por $\mathrm{H}$. Freeman Mathews, director de la Office of European Affairs, a Adolf Berle, importante miembro del grupo de asesores del presidente Roosevelt:

I can see no possible advantage in approving the OSS proposal to send Mr. De Irala to France. If the object is to get political intelligence concerning France, I think that we can soon obtain it through our own people, but if not, if would certainly seem preferable to send an American for the purpose. If the object is to obtain information with regard to Spain and the attitude of the Spanish Basques toward the Spanish Government, I think our Embassy and Consulates are fully capable of doing the job, and if not, I certainly would not favor sending a Basque. He would be, so to speak, an interested party, and I can see no American interest in promoting any plans for a Basque Republic at this stage $!^{49}$.

Las reticencias de algunos funcionarios estadounidenses no impidió que Irala se trasladara a Francia para actuar de enlace entre la OSS y los Servicios en misiones de sabotaje diseñadas por los estadounidenses para combatir los últimos reductos nazis en la región francesa de Aquitania.

No obstante, a las discrepancias generadas dentro de las agencias estadounidenses por el control de los proyectos de colaboración entre el SVI y la OSS, se debe sumar la mala reputación que esta última tuvo dentro de Estados Unidos. El Pentágono le negó el acceso a las comunicaciones interceptadas a Alemania y Japón, de importante valor informativo, porque la mayoría de la oficialía militar consideraba que un servicio de inteligencia civil independiente como el de Donovan, con acceso directo a Roosevelt, podía convertirse en un elemento peligroso para la seguridad de Estados Unidos, máxime cuando la mayoría de los agentes de la OSS eran -para ellos- de lo más burdo y estaban mal entrenados ${ }^{50}$. Pese a la negativa, la agencia de Donovan no se detuvo y buscó financiación para el proyecto de colaboración con los vascos a través de vías poco ortodoxas. Spencer Phenix, agente encargado de los asuntos relacionados con la Península Ibérica en Washington, propuso que los gastos de este proyecto fueran pagados a través de John Hughes, jefe de las oficinas de Nueva York, sin el conocimiento del Departamento de Estado, cada vez más reticente a aprobar partidas específicas de fondos especiales para misiones de la OSS ${ }^{51}$.

Tras la liberación de París en el verano de 1944, una gran mayoría del exilio vasco y español pensó que si las tropas aliadas avanzaban hacia la frontera española muy posiblemente intervendrían en España para acabar con Franco. El lehendakari Aguirre había puesto grandes esperanzas en Estados Unidos y el resto de los Aliados, pues al fin y al cabo -según su perspectiva-, tanto el Gobierno vasco en el exilio como las organizaciones nacionalistas vascas que lo apoyaban habían actuado correctamente, mostrándose dispuestos a colaborar en todo aquello -principalmente labores de espionaje y

\footnotetext{
48 NARA, "Carta de Spencer Phenix a William J. Donovan", sin lugar, 25-11-1944, RG 226, OSS Files, Entry 210, Box 391, WN 7169.

49 NARA, "Carta de William A. Kimbel a Spencer Phenix", sin lugar, 27-09-1944, RG 226 OSS files, Entry 210, Box 300, WN 12813, Leg. C12813/008.

${ }^{50}$ Tim WEINER, Legado de cenizas. Historia de la CIA, Barcelona: Debate, 2008, pp. 30 y ss.

${ }^{51}$ NARA, "Carta de Spencer Phenix a William J. Donovan", sin lugar, 25-11-1944, RG 226, OSS Files, Entry 210, Box 391, WN 7169.
} 
propaganda- que permitiera a los Aliados ganar la guerra contra el Eje. Según el lehendakari, si triunfaba Estados Unidos, líder del "bloque democrático", país cuyo presidente había avalado "la doctrina de libertad nacional de los pueblos", el Gobierno de Euzkadi tendría mayores oportunidades de restablecerse en territorio peninsular tan pronto como Franco fuera apartado del poder ${ }^{52}$.

\section{A MODO DE CONCLUSIÓN. LA NO-INTERVENCIÓN DE EEUU EN ESPAÑA}

Como se ha señalado, aunque algunos funcionarios del Departamento de Estado como Mathews se negaran a colaborar con los vascos y se mostraran reticentes a las operaciones conjuntas OSS-SVI, a la altura de octubre de 1944 Aguirre estaba convencido de que en España se llevaría a cabo un cambio de régimen patrocinado por los estadounidenses. Apenas unas semanas antes, la Agrupación de Guerrilleros Españoles, una organización impulsada por el PCE con el objetivo de agrupar fuerzas para luchar contra el franquismo, había iniciado la Operación Reconquista de España, una incursión de tropas de asalto del maquis -guerrilla antifranquista- que, aprovechando los últimos enfrentamientos contra los nazis en el sur de Francia, había entrado en España a través del valle de Arán, con la intención de instaurar un Gobierno republicano de carácter provisional en la zona ${ }^{53}$. Así valoró el presidente vasco tal intervención:

La revolución de España se producirá en un futuro muy cercano, creo que dentro de los próximos meses se registrarán grandes acontecimientos que remodelarán la paz en la Península lbérica [...]. Me mantengo en contacto con mi pueblo en España, así como con los catalanes y con otros líderes españoles, y sé que los preparativos revolucionarios se encuentran muy avanzados [...]. Los refugiados deben unirse, es de esperar que el rápido giro de los acontecimientos inducirá a ciertos grupos de refugiados discordantes a abandonar el faccionalismo y restablecer la unidad que piden tanto los maquis como el movimiento clandestino español [...]. Abogamos por consiguiente por una federación aún mayor, por una federación íbera que incluya no solo a todos los grupos nacionales dentro de España, sino de ser posible, también a Portugal. Esa sería la solución más saludable de los problemas de la Península íbera, pero debe ser alcanzada por medios espirituales y democráticos y no por la fuerza $a^{54}$.

Aguirre contaba con información privilegiada gracias a las entrevistas que había mantenido con Phenix, quien se había dirigido a Donovan en varias ocasiones para sugerirle que la OSS apoyara un movimiento clandestino dentro de España, que facilitara una transición pacífica hacia formas democráticas de gobierno. La inestabilidad del régimen de Franco era un hecho pero, si no se llevaban a cabo los pasos apropiados, la situación podría desembocar en el caos, la guerra civil y la anarquía. El Gobierno de Estados Unidos -explicó Phenix-, a tenor de su prestigio, poder e influencia, tenía cierta responsabilidad con los movimientos democráticos españoles y no podía permitir que estos perecieran

\footnotetext{
52 José Antonio Aguirre, "Misión de América", Euzko Deya México, no 25, p. 3, 1-3-1944. Id., "El derecho de los pueblos al gobierno propio", Euzko Deya México, no 33, p. 2, 1-7-1944.

53 José Luis CERVERO, "El maquis invade el valle de Arán: la operación "reconquista de España por los comunistas Monzón y López Tovar, es abortada", en Juan Carlos LAVIANA; Daniel ARJONA y Silvia FERNÁNDEZ (coords.), La liberación de París anima al maquis a "reconquistar" España: 1944, Madrid: Unidad Editorial, 2006, pp. 7-25. Joan ESTRUCH, El PCE en la clandestinidad (1939-1956), Madrid: Siglo XXI, 1982, p. 92 y ss. Andrés SOREL, La guerrilla antifranquista. La historia del maquis contada por sus protagonistas, Tafalla: Txalaparta, 2002. Ferrán SÁNCHEZ, Maquis a Catalunya: De la invasió de la Vall D’Aran a la Mort Del Caracremada, Lleida: Pagès Editors, 1999.

54 "Declaraciones del Presidente Aguirre a la International News Service", Euzko Deya México, no 41, p. 6, 1-111944.
}

HISPANIA NOVA., 15 (2017), págs. 172-190

DOI. https://doi.org/10.20318/hn.2017.3485 
bajo el yugo franquista o bajo el comunista. En el caso de que no se llegara a una solución pacífica concluyó el estadounidense-, la OSS debía preparar y presentar un programa de intervención dentro de España que aupara a un Gobierno de carácter democrático. Por la amistosa relación mantenida por la OSS con la delegación vasca, a efectos de colaboración informativo-propagandística -continuó Phenixel grupo de Aguirre era el adecuado para cooperar en el establecimiento de la democracia en España. De hecho, el presidente vasco ya se había ofrecido a liderar el movimiento pro-democrático e incluso había enviado instrucciones a los miembros de su gabinete para que apoyaran los esfuerzos del republicano católico y conservador Miguel Maura para que reorganizara y presidiera el Gobierno republicano. En un memorándum que dirigió a Donovan en diciembre de 1944, Phenix insistió en que si la OSS se decidía por apoyar un movimiento pro-democrático en España, los vascos eran los más apropiados para garantizar una transición pacífica:

We thus have ready to hand a strong a functioning Basque organization composed of energetic, courageous and competent people unselfishly devoted to the cause of democratic government and alive to the dangers of Communistic machinations and we believe that with appropriate support and guidance this group can play a possibly decisive role in the present critical chapter of Spanish history ${ }^{55}$.

Sobre la base de la colaboración mantenida entre la OSS y los vascos, gracias a la cual se estableció una red informativa a lo largo de los Pirineos sobre actividades enemigas que se llevaban a cabo en Sudamérica, se construyó una atmósfera de confianza mutua. Spencer Phenix señaló a Donovan que, si la Casa Blanca y el Departamento de Estado decidían tomar medidas para establecer en España un régimen democrático que sustituyera la dictadura de Franco, la OSS y la organización vasca podían prestar una ayuda incalculable. Por tanto, el representante de esta agencia en la Península debía ser instruido para que planificara el cambio de régimen político junto al nuevo embajador de Estados Unidos en España, Norman Armour, cuyas decisiones deberían ser aprobadas por el presidente y el secretario de Estado a fin de evitar que Estados Unidos se viera involucrado en compromisos diplomáticos. Aunque no existieran garantías de que en España se pudiera llevar a cabo un cambio político pacífico Phenix advirtió de "the consequences of a hands-off policy could be so disastrous not only for Spain but also for the cause of democracy and liberty in other parts of the world, that the seizing of even a forlorn chance would seem worthwhile" ${ }^{56}$.

Muchas de las sugerencias y advertencias contenidas en este memorándum de Phenix coincidieron con las apreciaciones de John Hickerson, subdirector de la Office of European Affairs. Este señaló a James C. Dunn, subsecretario de Estado para los Asuntos de Europa, África y Oriente Próximo, que no esperara relaciones satisfactorias entre Estados Unidos y España mientras Franco fuera el jefe del Estado y Falange continuara estando cerca del poder. Era "time to change in Spain", pero ni Estados Unidos ni la OSS debían implicarse directamente sino impulsar la acción de aquellos elementos peninsulares amigos de la democracia como los contactos vascos de Spencer Phenix ${ }^{57}$. Con todo, la no

\footnotetext{
55 NARA, "Memorándum de Spencer Phenix para William Donovan", Washington DC, 16-12-1944, RG 59, State Department, Box 5234, Leg. 852.00/12-1944.

${ }^{56}$ Ibídem

${ }^{57}$ NARA, “Carta de John Hickerson a James C. Dunn”, sin lugar, 3-1-1945, RG 59, State Department, Box 5234, Leg. 852.00/12-1944.
}

HISPANIA NOVA., 15 (2017), págs. 172-190

DOI. https://doi.org/10.20318/hn.2017.3485 
interferencia directa en los asuntos políticos propios de los españoles fue la máxima que dominó dentro del Departamento de Estado, que siguió la estela de la política practicada desde Londres ${ }^{58}$.

Esta idea fue apoyada por diplomáticos como Paul T. Cullbertson, convencido de que no había ningún elemento dentro de la oposición política española (incluidos los vascos) que pudiera desembocar en la formación de un gobierno alternativo al franquismo. Por tanto aconsejó a la OSS que no se inmiscuyera en iniciativas contra Franco para evitar, en caso de que el plan fracasara y fuera descubierto por las autoridades españolas, inconveniencias diplomáticas para Estados Unidos ${ }^{59}$. Esta opinión fue avalada por los informes enviados por William J. Gallman -encargado de negocios de la embajada de Estados Unidos en Londres- al Departamento de Estado, que indicó que el único interés de los nacionalistas vascos, al igual que el de catalanes y gallegos, era el reemplazo de un gobierno español centralista por el de una federación de naciones, y que su implicación en el restablecimiento de la democracia española y su lucha contra el comunismo dependía del grado de independencia que obtuvieran estas. Según adujo este diplomático, la política de apoyo a la restauración de la República española respondía al interés por obtener una contraprestación -el autogobierno- para los nacionalismos vasco, catalán y gallego, aunque esto supusiera que en un futuro tuvieran que entenderse con el sector comunista liderado por el socialista Juan Negrín ${ }^{60}$. No ayudó a cambiar esta percepción las cartas interceptadas por la inteligencia estadounidense a otro de los líderes socialistas, Indalecio Prieto, que definió la actuación política del Gobierno vasco como "de franco carácter separatista" y carente de apoyo en el seno del exilio socialista en América ${ }^{61}$. En buena medida, la declaración que meses después, en marzo de 1945, publicó el Gobierno vasco en Nueva York, apoyando la causa democrática española y promoviendo la unidad entre los republicanos españoles, a quienes

\footnotetext{
${ }^{58}$ Florentino PORTERO, “El régimen franquista y Estados Unidos. De enemigos a aliados", en Lorenzo DELGADO y María Dolores ELIZALDE: España y Estados Unidos en el siglo XX, Madrid: CSIC, 2005, p. 142 y ss. Alejandro PIZARROSO: Diplomáticos, propagandistas y espías, Estados Unidos y España en la Segunda Guerra Mundial: información y propaganda, CSIC, Madrid, 2009, p. 140 y ss. Joan E. Garcés, Soberanos e intervenidos. Estrategias globales, americanos y españoles, Madrid: Siglo XXI, 1996, p. 378 y ss.

59 NARA, “Carta de Paul T. Culbertson a James C. Dunn", sin lugar, 28-12-1944, RG 59, State Department, Box 5234, Leg. 852.00/12-1944.

60 NARA, Informe de investigación confidencial titulado "Spanish Opposition Forces", realizado por el Departamento de Política Exterior, y enviado por William J. Gallman al secretario de Estado de EEUU, Edward Stettinius Jr., sin lugar, 31-1-1945, RG. 59 State Department, Box 6334, Leg. 852.00/1-3145. Hay que recordar que el socialismo español en el exilio estaba muy dividido. Durante la Guerra Civil, Negrín se había apoyado en el Partido Comunista para tratar de llevar a cabo su política de resistencia a ultranza, opuesta a sectores, como el de Indalecio Prieto, partidarios de una mediación. Julio Aróstegui: “Caballero, Negrín, Prieto, Besteiro, cuatro socialistas ante el problema comunista en la guerra civil", en Francisco MORENTE: España en la crisis europea de entreguerras: república, fascismo y guerra civil, Madrid: Catarata, 2011, pp. 179-210. Abdón MATEOS: "Prieto contra Negrín 1938-1939", en Abdón MATEOS (coord.): Indalecio Prieto y la política española, Madrid: Editorial Pablo Iglesias, 2008, pp. 193-230. José Carlos GIBAJA: “Indalecio Prieto y la reconstrucción del socialismo español en el exilio", en Abdón MATEOS Indalecio Prieto, op. cit., pp. 231-280. Enrique MORADIELLOS: Negrín, Barcelona: Península, 2006. Josep SÁNCHEZ CERVELLÓ: La Segunda República en el exilio (1939-1977), Planeta, Barcelona, 2011, pp. 31 y ss.

${ }^{61}$ NARA, "Carta de Indalecio Prieto a Paulino Gómez Beltrán”, México D.F., 15-2-1945, RG 226, OSS Files, Entry 108, Box 260, Leg. RF-192.
} 
alentaba a la apertura de conversaciones para llegar a un acuerdo que ayudase a acabar con la dictadura franquista, fue una réplica inconsciente a las afirmaciones del dirigente socialista ${ }^{62}$.

Como hemos visto, este decidido apoyo de Phenix a la idea de promover activamente la causa democrática en España confirma que, como ya señaló Ludger Mees, la estrategia gradualista impulsada por el lehendakari, de buscar una solución al problema español como forma de devolver al Gobierno de Euzkadi a territorio vasco, fue bien recibida por algunas esferas de poder estadounidenses, en la medida en que la organización política vasca se presentó como un instrumento político efectivo para echar a Franco y establecer un nuevo régimen político paritariamente contrario al fascismo y al comunismo ${ }^{63}$. Sin embargo, el tiempo demostró poco después a los vascos que la bicefalia imperaba en el Gobierno de Estados Unidos. Mientras que un sector fue ideológicamente cercano y favorable a apoyar la alternativa del exilio político vasco y español, otro, el mayoritario, impuso una praxis que puso distancia de por medio con este, primando el mantenimiento del statu quo con el objetivo de proteger sus intereses. La Guerra Fría había comenzado.

\footnotetext{
${ }^{62}$ Archivo Histórico del Nacionalismo Vasco (AHNV), "Declaración del Gobierno vasco", Nueva York, 2-3-1945, PNV NAC EBB K.304 C.9.

${ }^{63}$ Ludger MEES, El profeta pragmático, op. cit., p. 79.
} 